\title{
Flow Conditions in the Intracranial Aneurysm Lumen Are Associated with Inflammation and Degenerative Changes of the Aneurysm Wall
}

(D). Cebral, (DE. Ollikainen, (D)B.J. Chung, (D) F. Mut, (D) V. Sippola, (D)B.R. Jahromi, (D)R. Tulamo, (D). Hernesniemi, (D) M. Niemelä, (D) A. Robertson, and (D). Frösen

\begin{abstract}
BACKGROUND AND PURPOSE: Saccular intracranial aneurysm is a common disease that may cause devastating intracranial hemorrhage. Hemodynamics, wall remodeling, and wall inflammation have been associated with saccular intracranial aneurysm rupture. We investigated how saccular intracranial aneurysm hemodynamics is associated with wall remodeling and inflammation of the saccular intracranial aneurysm wall.
\end{abstract}

MATERIALS AND METHODS: Tissue samples resected during a saccular intracranial aneurysm operation (11 unruptured, 9 ruptured) were studied with histology and immunohistochemistry. Patient-specific computational models of hemodynamics were created from preoperative CT angiographies.

RESULTS: More stable and less complex flows were associated with thick, hyperplastic saccular intracranial aneurysm walls, while slower flows with more diffuse inflow were associated with degenerated and decellularized saccular intracranial aneurysm walls. Wall degeneration $(P=.041)$ and rupture were associated with increased inflammation (CD45,$+ P=.031)$. High wall shear stress $(P=.018)$, higher vorticity $(P=.046)$, higher viscous dissipation $(P=.046)$, and high shear rate $(P=.046)$ were associated with increased inflammation. Inflammation was also associated with lack of an intact endothelium $(P=.034)$ and the presence of organized luminal thrombosis $(P=.018)$, though overall organized thrombosis was associated with low minimum wall shear stress $(P=.034)$ and not with the flow conditions associated with inflammation.

CONCLUSIONS: Flow conditions in the saccular intracranial aneurysm are associated with wall remodeling. Inflammation, which is associated with degenerative wall remodeling and rupture, is related to high flow activity, including elevated wall shear stress. Endothelial injury may be a mechanism by which flow induces inflammation in the saccular intracranial aneurysm wall. Hemodynamic simulations might prove useful in identifying saccular intracranial aneurysms at risk of developing inflammation, a potential biomarker for rupture.

ABBREVIATIONS: $\max =$ maximum; OSI = oscillatory shear index; $\mathrm{sIA}=$ saccular intracranial aneurysm; $\mathrm{VO}=$ vorticity; WSS = wall shear stress

S accular intracranial aneurysm (sIA) is a relatively common disease (estimated prevalence, $2 \%-3 \%^{1}$ ), which is often undiagnosed because of a lack of symptoms but may cause a devastating intracranial hemorrhage. Some patients die immediately after sIA rupture, and of those patients who make it to the hospital after

\footnotetext{
Received May 10, 2016; accepted after revision July 22.

From the Bioengineering Department (J.C., B.J.C., F.M.), Volgenau School of Engineering, George Mason University, Fairfax, Virginia; Neurosurgery Research Group (E.O., V.S., B.R.J., R.T., J.H., M.N., J.F.), Biomedicum Helsinki and Helsinki University Central Hospital, Helsinki, Finland; Mechanical Engineering and Materials Science and Department of Bioengineering (A.R.), Swanson School of Engineering, University of Pittsburgh, Pittsburgh, Pennsylvania; Department of Vascular Surgery (R.T.), Helsinki University Central Hospital, Helsinki, Finland; and Department of Neurosurgery and Hemorrhagic Brain Pathology Research Group (J.F.), Neurocenter, Kuopio University Hospital, Kuopio, Finland.

This work was supported by National Institutes of Health grant R21 NS080031-01A1, Helsinki University Central Hospital research grants TYH2012201 and TYH2014236, Kuopio University Hospital research grants, Helsinki Biomedical Graduate Program funding, the Orion Research Foundation, and the Finnish Medical Foundation.
}

sIA rupture, $27 \%$ die despite neurosurgical intensive care. ${ }^{2}$ Because of the sinister outcome of sIA rupture, many diagnosed unruptured sIAs are treated to prevent rupture. However, many, if not most, sIAs do not rupture during life-long follow-up. ${ }^{3}$ Moreover, currently, sIA rupture can be prevented only by invasive procedures (endovascular or microsurgical occlusion) with a significant risk of morbidity and even mortality. ${ }^{4}$ It is therefore extremely important to distinguish rupture-prone sIAs from those that will never rupture, especially because unruptured sIAs are being diagnosed with increasing frequency due to improved access to MR imaging and other imaging studies of the brain.

\footnotetext{
Please address correspondence to Juhana Frösen, MD PhD, Department of Neurosurgery and Hemorrhagic Brain Pathology Research Group, Puijonlaaksontie 2, Kuopio University Hospital 70029, Kuopio, Finland; e-mail: juhana.frosen@kuh.fi

- Indicates open access to non-subscribers at www.ajnr.org

三 Indicates article with supplemental on-line tables.

http://dx.doi.org/10.3174/ajnr.A4951
} 
An aneurysm wall ruptures when the wall strength is exceeded by the mechanical stress imposed on it. Wall strength depends on wall structure, which is dependent on the cellular and extracellular composition of the sIA wall. The characteristics of the cellular and extracellular composition of the ruptured or rupture-prone sIA wall, mainly damage to the endothelium, inflammation, and loss of mural cells, have been previously reported by us and others. ${ }^{5-8}$ The cause of these histologic changes, which we refer to as "degenerative remodeling" remains unknown.

We have previously described the potential mechanism by which nonphysiologic flow conditions can trigger degenerative remodeling of the sIA wall via damage to the endothelium. ${ }^{10,11}$ In addition, we and others have previously shown that flow and wall shear stress (WSS) differ in ruptured and unruptured sIAs ${ }^{12-14}$ and that these flows are associated with changes in sIA morphology. ${ }^{15}$ The association of flow and WSS with sIA rupture suggests that they affect sIA wall structure, the main determinant of rupture risk.

Here we studied the possible association of hemodynamics with the histologic changes of the sIA wall. We focused especially on wall degeneration, inflammatory cell infiltration, and damage to the endothelium.

\section{MATERIALS AND METHODS}

\section{Patients and Tissue Samples of Intracranial Aneurysms}

Patients with intracranial aneurysms that underwent surgical clipping were considered for the study. Twenty patients with preoperative 3D imaging necessary for computational fluid dynamics analysis were included (On-line Table 1). During the surgical intervention, after placement of the clip, a tissue sample was harvested from the aneurysm dome for histology and immunohistochemical analysis. The size of the tissue sample excised from the aneurysm wall varied depending on the size of the aneurysm and how the aneurysm was clipped (Fig 1). Of these 20 patients, 10 were included in a prior histopathologic study by our group. ${ }^{17}$ The study was approved by the ethics committee of Helsinki University Central Hospital, and patients gave informed consent to participate to the study.

\section{Histology and Immunohistochemistry}

The 20 aneurysm tissue samples underwent immunostaining against CD45 (pan-leukocyte marker, clone 2B11+PD7/26, dilution 1:400; DAKO, Glostrup, Denmark; Fig 1). The degree of inflammation in the sIA wall was quantified by calculating the number of CD45-positive cells per standardized surface area $(0.613$ $\mathrm{mm}^{2}$ ) under a $\times 20$ magnification from 1 to 3 hotspots in the intracranial aneurysm wall. Degenerative remodeling of the sIA wall was scored from hematoxylin-eosin staining by using a previously described scale based on the cellular composition of the sIA wall and the structure of the sIA wall extracellular matrix. ${ }^{6}$ This scale is associated with the rupture rate. ${ }^{6}$ The presence of fresh or organized luminal thrombus was assessed from hematoxylin-eosin staining. The presence of endothelium on the luminal surface was scored from CD31 immunostainings according to the morphology of the cell and positivity to CD31 (Fig 1, CD31 data available for only a subset of 10 aneurysms) as described previously by Ollikainen et al. ${ }^{17}$
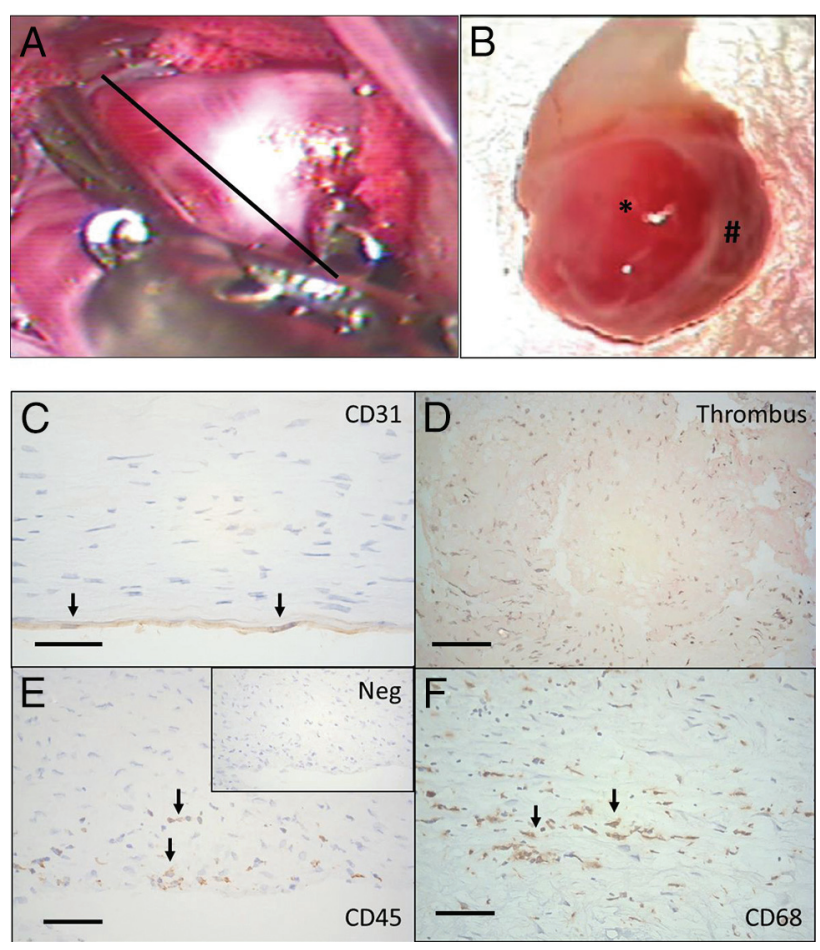

FIG 1. Tissue samples were harvested after aneurysm clipping by cutting through the aneurysm dome distal to the clip, as demonstrated in $A$ (black line represents the estimated site of cut). $B$, The tissue samples obtained varied significantly in size (from small, approximately $2 \times 1 \mathrm{~mm}$ tissue samples to half domes with a $>10-\mathrm{mm}$ radius). In addition to variation in size, many of the aneurysm samples had local variation in the appearance of the wall (B) with translucent areas (asterisk) and thicker wall regions (hash tag). C, In histology, endothelium (CD31+ luminal cells with endothelial cells morphology) was present in only $3 / 10$ of the studied aneurysm walls. $D, \ln 8 / 20$ aneurysms, the luminal surface was instead covered by thrombus, and in $7 / 20$ aneurysms, some degree of thrombus organization was observed (hematoxylin-eosin staining). E, Loss of endothelium and organizing luminal thrombosis were associated with inflammatory cell infiltration $(\mathrm{CD} 45+)$ in the aneurysm wall. $F$, Many of the inflammatory cells were macrophages (CD68+). Immunostaining protocol for CD68 is described by Ollikainen et al. ${ }^{17}$ Scale bars represent a $50-\mu \mathrm{m}$ scale. Negative control for immunostaining is given for CD45 staining (Neg). The black arrows indicate positive cells.

\section{Computational Flow Analysis}

Image-based computational fluid dynamics models were constructed from preoperative 3D CT angiography images. ${ }^{18}$ CTA images consisted of approximately 250 axial sections with $512 \times$ 512 pixels and a typical voxel resolution of $0.4 \times 0.4 \times 0.6 \mathrm{~mm}$. Pulsatile flow simulations were performed by solving the $3 \mathrm{D}$ incompressible Navier-Stokes equations by using finite elements on unstructured grids. ${ }^{19}$ The computational meshes had a resolution of $200 \mu \mathrm{m}$ and contained between 2 and 5 million elements. Inflow boundary conditions were prescribed by using the Womersley velocity profile $\mathrm{e}^{20}$ and flow waveforms derived from flow measurements in healthy subjects. ${ }^{21,22}$ Inflow rates were scaled with the inlet area according to the Murray law, ${ }^{23}$ and mean flows ranged from approximately 1.2 to $4.1 \mathrm{~mL} / \mathrm{s}$. Outflow conditions were prescribed by splitting the outflow rates according to the principle of minimum work (Murray law). ${ }^{23}$ Vessel walls were assumed rigid, and blood was approximated as a Newtonian fluid with a density of $\rho=1.0 \mathrm{~g} / \mathrm{cm}^{3}$ and a viscosity of $0.04-\mu \mathrm{m}$ poise. Numeric solutions were obtained for 2 cardiac cycles by using 100 
time-steps per cycle. Flow fields corresponding to the second cycle were saved for analysis.

To characterize the aneurysm hemodynamic environment, we computed a number of flow variables over the aneurysm region (On-line Table 2). Exact mathematic definitions of these quantities are given by Mut et al. ${ }^{24}$ Our choice of mesh resolution and time-step size has been shown to be adequate for accurate quantification of the spatial and time-averaged quantities considered in this work. ${ }^{25}$

\section{Geometric Analysis}

The geometry of the aneurysm (ie, its size and shape) was characterized by automatically computing several geometric variables by using 3D vascular reconstruction (On-line Table 3). Definitions of these quantities and a physical explanation of their meaning can be found in $\mathrm{Ma}$ et $\mathrm{al}^{26}$ and Raghavan et al. ${ }^{27}$

\section{Statistical Analysis}

Because our hypothesis was that it is the nonphysiologic WSS (whether too high or too low) but not the physiologic WSS that triggers the degenerative remodeling and inflammation of the sIA wall, ${ }^{10}$ we classified our aneurysms accordingly into groups of low, mid-, or high WSS instead of directly comparing WSS with wall remodeling or inflammation. Because there is no clear definition for what constitutes the physiologic range of WSS, especially in a saccular intracranial aneurysm, we classified our aneurysms according to the median of the time and sac-averaged WSS magnitude $\left(8.9\right.$ dyne $\left./ \mathrm{cm}^{2}\right)$ and its SD $\left(12.6\right.$ dyne $\left./ \mathrm{cm}^{2}\right)$. These groups were the following: 1) low WSS: aneurysms with WSS $<$ $\mathrm{WSS}_{0}$; 2) mid-WSS: aneurysms with $\mathrm{WSS}_{0}<\mathrm{WSS}<\mathrm{WSS}_{1}$; and 3) high WSS: aneurysms with WSS $>\mathrm{WSS}_{1}$. The $\mathrm{WSS}_{0}$ and WSS were calculated as one-half and twice the WSS median over the 20 aneurysms $\left(\mathrm{WSS}_{0}=4.5 \mathrm{dyne} / \mathrm{cm}^{2}\right.$ and $\left.\mathrm{WSS}_{1}=18.0 \mathrm{dyne} / \mathrm{cm}^{2}\right)$. The number of inflammatory cells was then compared as a continuous variable among these 3 groups.

Next, to compare the hemodynamic and geometric characteristics between aneurysms with little inflammation or substantial inflammation, the samples were classified as the following: group 1: little inflammation if the number of CD45+ was less than the median of CD45 + counts for all samples, and group 2: substantial inflammation if the number of CD45 + was larger than the median of CD45 + counts for all samples. The hemodynamic and geometric indices were then compared as continuous variables between the groups.

The flow-related parameters are given as time-averaged (over systole-diastole) and space-averaged (over aneurysm volume including the neck) values, unless otherwise defined. To study the potential effect of focal variations in the wall shear stress distribution and in inflammation, we categorized both according to their heterogeneity and compared the scores. Flow was defined as homogeneous if the mean over time of the spatial SD/mean over time of the spatial average was $<0.5$ or the average was $<3$ dyne/ $\mathrm{cm}^{2}$. All other flows were defined as heterogeneous. Heterogeneity of the CD45 count was considered in the context of the heterogeneity in the reported values for CD45 hotspots. The number of hotspots ranged from 1 to 3. Samples with only 1 spot were removed from analysis; 18 were assessed for wall heterogeneity. The wall was considered homogeneous if all values in the sample were less than a threshold of 10 or greater than a threshold of 200. For those between these threshold values, a value of (maximumminimum) $/ \mathrm{SD}<1.0$ was considered homogeneous.

Overall, proportions and frequencies were calculated for categoric variables; and for continuous variables, median and range were used because the distributions of the continuous variables deviated from normal distribution. The Fisher exact test was used to compare categoric variables. The nonparametric Spearman rank correlation test was used for correlations between 2 continuous variables; and the Mann-Whitney $U$ test, for comparison of continuous and categoric variables. The Mann-Whitney $U$ test was used as a one-sided test due to our limited statistical power ( $\leq 20$ cases). Statistical analyses were performed by using Python scripts and the SciPy package (http://www.scipy.org/) or SPSS statistical software (IBM, Armonk, New York). $P$ values $<.05$ were considered significant.

\section{RESULTS}

\section{Factors Associated with Wall Degeneration and Rupture: Inflammation}

In this series, flow-related hemodynamic variables did not significantly differ between ruptured and unruptured sIAs (On-line Table 3). Of the studied geometric variables, only the ellipticity index $(P=.047)$ and Gaussian curvature $(P=.047)$ were associated with rupture (On-line Table 4).

Compared with sIAs with walls that have a histology most resembling the composition of the normal arterial wall (A type, $n=2$ ), sIAs with both thick walls and hyperplasia of mural cells (B-type walls, $n=10$ ) as well as degenerated walls with loss of mural cells (C type, $n=8$ ) had less oscillatory flows (lower oscillatory shear index [OSI] and maximum oscillatory shear index [OSImax], Table 1). Interestingly, thick hyperplastic walls were also associated with simpler and more stable flows (lower corelen, vortex core line length, a measure of flow complexity; and lower podent, proper orthogonal decomposition entropy, a measure of flow instability; Table 1), whereas loss of mural cells was associated with slower and more diffuse inflows (Table 1).

Degenerated walls characterized by loss of mural cells were associated with increased infiltration of inflammatory cells (wall types B versus $C, P=.011$; A versus $C, P=.041$ ). In addition to wall degeneration, inflammation was associated with rupture $(P=.031$, Fig 2A).

\section{Factors Associated with Aneurysm Wall Inflammation: High Flow Conditions and Endothelial Damage}

Inflammation was associated with WSS (Figs $2 B$ and 3). The degrees of wall inflammation (CD45+ cells) in each WSS group are presented in Fig $2 B$. Aneurysms with high WSS $(n=4)$ had larger numbers of inflammatory cells than sIAs with mid-WSS levels ( $n=9, P=.018)$. Also sIAs with low WSS $(n=7)$ had somewhat higher numbers of inflammatory cells than sIAs with mid-WSS levels, though the difference did not reach statistical significance. Other flow characteristics associated with increased inflammation were higher vorticity (VO, $P=.046)$, higher viscous dissipation $(P=.046)$, and higher shear rate $(P=.046)$. Heterogeneity in the distribution of WSS across a single aneurysm sac was associ- 
Table 1: Associations between flow characteristics and composition of the sIA wall ${ }^{\mathrm{a}}$

\begin{tabular}{|c|c|c|c|c|}
\hline \multirow{2}{*}{$\begin{array}{c}\text { Hemodynamic } \\
\text { Variable }\end{array}$} & \multicolumn{4}{|c|}{ Wall Type } \\
\hline & A & B & C & $P$ Values \\
\hline $\mathrm{ICl}$ & $2.24(1.92-2.56)$ & $0.62(0.11-2.91)$ & $0.36(0.22-1.67)$ & $\mathrm{p}_{\mathrm{AB}}=.119, \mathrm{p}_{\mathrm{AC}}=.04,{ }^{\mathrm{b}} \mathrm{p}_{\mathrm{BC}}=.334$ \\
\hline Q & $1.71(1.22-2.19)$ & $0.67(0.52-2.32)$ & $0.52(0.31-0.95)$ & $\mathrm{P}_{\mathrm{AB}}=.081, \mathrm{p}_{\mathrm{AC}}=.041,{ }^{\mathrm{b}} \mathrm{p}_{\mathrm{BC}}=.291$ \\
\hline Corelen & $5.56(4.31-6.81)$ & $1.48(0.00-3.11)$ & $0.46(0.03-4.80)$ & $\mathrm{p}_{\mathrm{AB}}^{\mathrm{AB}}=.021,{ }^{\mathrm{b}} \mathrm{p}_{\mathrm{AC}}=.088, \mathrm{p}_{\mathrm{BC}}=.476$ \\
\hline Podent & $0.55(0.37-0.74)$ & $0.24(0.13-0.54)$ & $0.19(0.13-0.44)$ & $\mathrm{p}_{\mathrm{AB}}=.034,{ }^{b} \mathrm{p}_{\mathrm{AC}}=.088, \mathrm{p}_{\mathrm{BC}}=.334$ \\
\hline OSImax & $0.47(0.46-0.49)$ & $0.36(0.26-0.46)$ & $0.28(0.10-0.44)$ & $\mathrm{P}_{\mathrm{AB}}=.034,{ }^{\mathrm{b}} \mathrm{P}_{\mathrm{AC}}=.041,{ }^{b} \mathrm{p}_{\mathrm{BC}}=.213$ \\
\hline OSI & $0.044(0.03-0.05)$ & $0.02(0.01-0.03)$ & $0.01(0.00-0.03)$ & $\mathrm{P}_{\mathrm{AB}}=.021,{ }^{\mathrm{b}} \mathrm{P}_{\mathrm{AC}}=.041,{ }^{\mathrm{b}} \mathrm{P}_{\mathrm{BC}}=.427$ \\
\hline
\end{tabular}

Note:- $\mathrm{ICl}$ indicates inflow concentration index; $\mathrm{Q}$, inflow rate into the aneurysm; corelen, vortex core line length, a measure of flow complexity; podent, proper orthogonal decomposition entropy, a measure of flow instability.

${ }^{a}$ Median and range are given for the hemodynamic variables in each wall type group, and corresponding $P$ values for comparisons between groups are given. $P_{A B}=P$ value $(A$ vs $B$ ), $P_{A C}=P$ value ( $\mathrm{vs} C$ ), $P_{B C}=P$ value ( $B$ vs $C$ ). Group $A$ is defined by an intact-looking wall with linearly organized smooth muscle cells and intact endothelium; group $B$, by smooth muscle cell hyperplasia; and group $\mathrm{C}$, by loss of smooth muscle cells and endothelial cells complemented by degeneration of the extracellular matrix and luminal thrombosis.

bignificant.

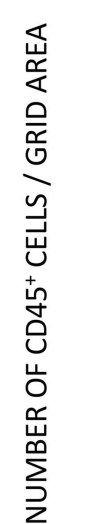

A
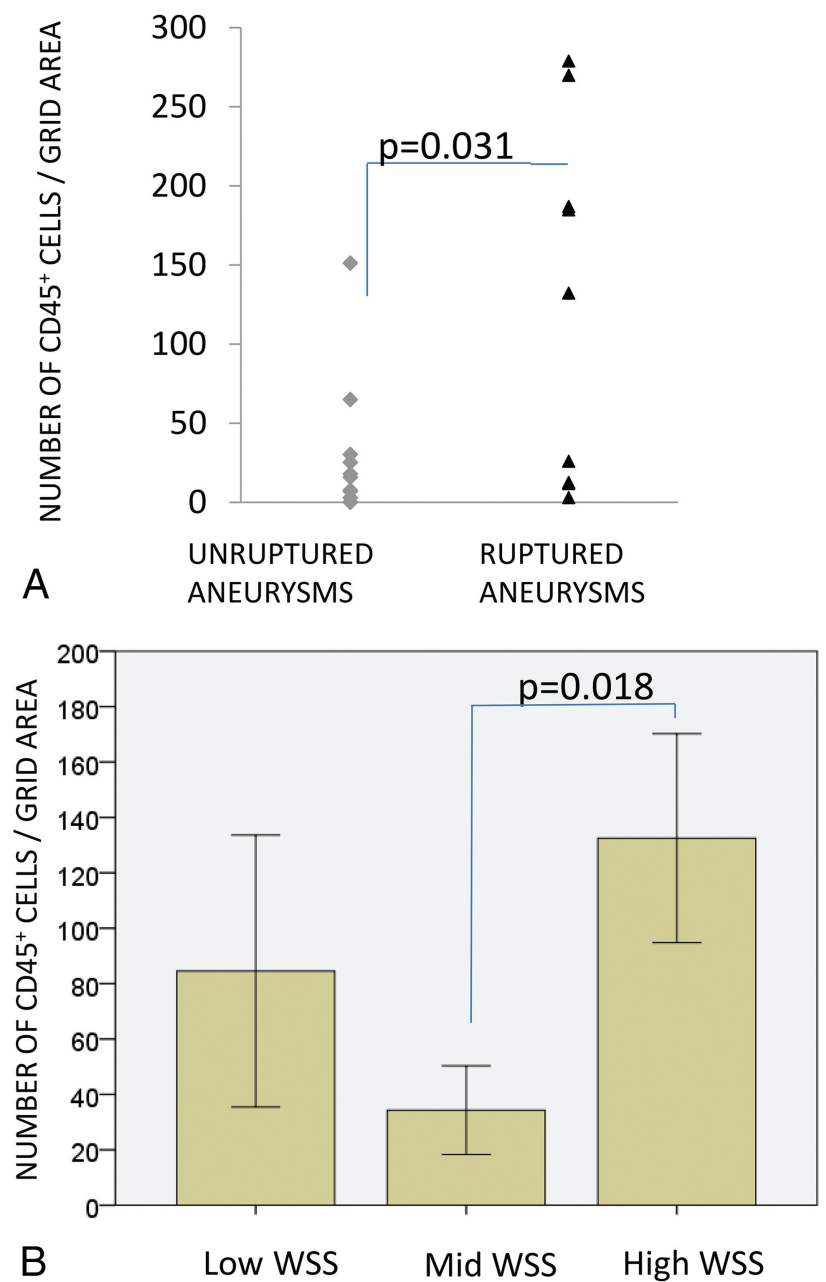

FIG 2. A, Infiltration of inflammatory cells $(C D 45+)$ associated with rupture and high mean wall shear stress. $B$, WSS grouping is as follows: mean WSS of $<4.5$ dyne $/ \mathrm{cm}^{2}=$ low; $4.5<$ mean WSS $<$ 18 dyne $/ \mathrm{cm}^{2}=$ mid; mean WSS $>18$ dyne $/ \mathrm{cm}^{2}=$ high. Bars display means, and error bars, standard error of mean.

ated with heterogeneity in the number of CD45 + cells across the wall ( $P=.035)$, suggesting that interactions leading to the association of high WSS and inflammation might happen focally at the wall. More inflammatory cells $(\mathrm{CD} 45+)$ were found in sIA walls that lacked an intact endothelium $(P=.034$, Table 2$)$, as well as in sIAs with organized thrombus $(P=.018)$, suggesting that
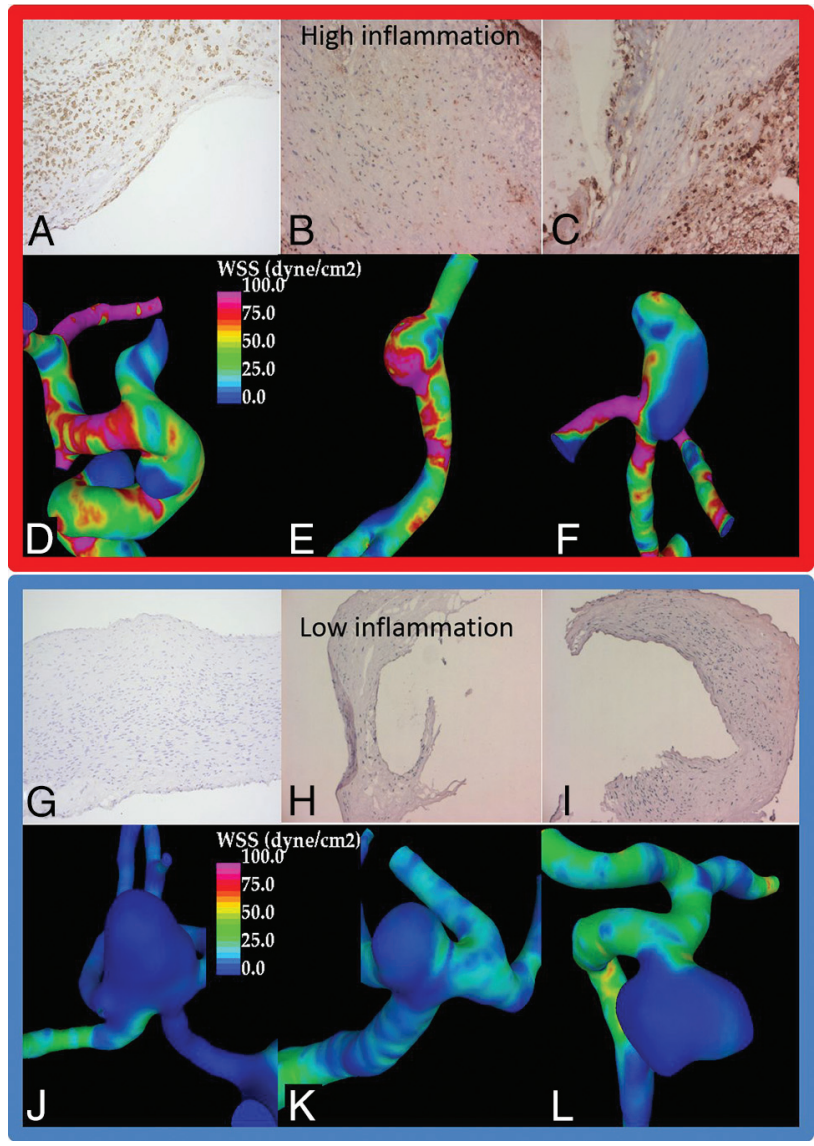

FIG 3. $A-F$, Three aneurysms from the high WSS group with substantial wall inflammation. $A-C, C D 45$ immunostaining and inflammatory cells in brown along with 3 aneurysms from the low WSS group (G-L) with little wall inflammation ( $-L$, negative CD45 immunostaining).

damage to the endothelium may mediate the association of flow and inflammation.

\section{Factors Associated with Endothelial Damage: High Flow Conditions}

Lack of an intact endothelium was associated with higher vorticity $(P=.034)$, higher viscous dissipation $(P=.020)$, and higher shear rate $(P=.034)$, similar to inflammation. In addition, lack of an endothelium was associated with lower OSImax $(P=.034)$, similar to wall remodeling (B- and C-type walls compared with A-type walls). In this series, the sIAs that lacked an intact endo- 
Table 2: Association of damage to the sIA wall endothelium (assessed through presence of CD31+ luminal cells with endothelial cell morphology) with wall inflammation (CD45+ cells), hemodynamics, and geometry of the sIA ${ }^{\mathrm{a}}$

\begin{tabular}{lccc}
\hline & \multicolumn{2}{c}{ Condition of the Endothelium } & \\
\cline { 2 - 3 } Variables & Intact & Damaged & $P$ Value \\
\hline Histology & & & \\
CD45+ cells & $13(7-8)$ & $30(12-279)$ & $.034^{\mathrm{b}}$ \\
Hemodynamics & & & \\
VO & $41.2(38.9-72.3)$ & $191.8(47.3-317.8)$ & $.034^{\mathrm{b}}$ \\
VD & $23.2(15.9-45.6)$ & $291.1(35.0-1027.6)$ & $.020^{\mathrm{b}}$ \\
SR & $28.2(27.7-51.3)$ & $129.1(41.5-231.9)$ & $.034^{\mathrm{b}}$ \\
OSImax & $0.30(0.04-0.41)$ & $0.18(0.01-0.47)$ & $.034^{\mathrm{b}}$ \\
Geometry & & & \\
Size & $15 \mathrm{~mm}(10-21 \mathrm{~mm})$ & $7 \mathrm{~mm}(4-12 \mathrm{~mm})$ & $.020^{\mathrm{b}}$ \\
Nsize & $9 \mathrm{~mm}(7-13 \mathrm{~mm})$ & $5 \mathrm{~mm}(4-9 \mathrm{~mm})$ & $.020^{\mathrm{b}}$ \\
UI & $0.13(0.04-0.13)$ & $0.25(0.10-0.35)$ & $.034^{\mathrm{b}}$ \\
EI & $0.24(0.24-0.24)$ & $0.26(0.25-0.30)$ & $.011^{\mathrm{b}}$ \\
\hline
\end{tabular}

Note:-VD indicates viscous dissipation; SR. shear rate; Nsize, neck size; UI, increased undulation; El, ellipticity index.

${ }^{a}$ Values are given as median and range.

b Significant.

thelium were smaller $(P=.020)$, had smaller necks $(P=.020)$, and had increased undulation $(P=.034)$ and ellipticity $(P=.011$, Table 2).

\section{DISCUSSION}

Degenerative wall remodeling, inflammation, and nonphysiologic flow conditions have been previously shown to be associated with sIA rupture. ${ }^{6,13,14,28}$ Although we and others have suggested possible mechanisms by which pathologic flow conditions may lead to degenerative wall remodeling, ${ }^{10,12,16,29}$ this is the first study that compares in vivo flow conditions with the histology of human intracranial aneurysms.

\section{Flow Conditions Associated with Degenerative Wall Remodeling and Inflammation}

We have previously shown that loss of mural smooth muscle cells leads to aneurysm growth and rupture in experimental models ${ }^{9}$ and is associated with rupture in human sIAs. ${ }^{30}$ The cause of smooth muscle cell loss and the degenerative remodeling in human aneurysms is still unknown, but inflammation of the sIA wall has been suggested as a potential cause of the degenerative remodeling that predisposes to rupture. ${ }^{31}$ Moreover, even if not the cause of wall degeneration, inflammation modulates the remodeling of the sIA wall. ${ }^{7,10}$

In this series, proliferative (hyperplasia) wall remodeling was associated with simple, stable flows, whereas degenerative remodeling (loss of cells and damage to the extracellular matrix) was associated with slower and diffuse inflows. Whether simple, stable flows are needed for the proliferative remodeling, whereas slower and diffuse inflows trigger degenerative processes leading to cell death in the sIA wall remains to be determined. Inflammation of the sIA wall was significantly associated with high flow conditions (Figs 3 and 4), but not with flow conditions that were associated with degenerative remodeling and loss of mural cells (C-type walls). This finding could suggest that flow patterns and hemodynamic stress may affect sIA wall remodeling, including mural smooth muscle cell proliferation and cell death, independent of wall inflammation. Moreover, it suggests that part of the inflammatory cell response in the sIA wall may be induced by high flow conditions, independent of the wall remodeling.

Flow-Mediated Endothelial Damage as the Trigger of Wall Remodeling and Inflammation?

Endothelial cells are sensors of WSS in the arterial wall. ${ }^{32}$ They contribute to the control of blood flow in the vessel by regulating muscle tone in the vessel wall and subsequently vessel diameter. ${ }^{32}$ Endothelial cells also maintain a very important barrier function between the bloodstream and the vessel wall ${ }^{32-34}$ and actively prevent luminal thrombosis. ${ }^{35}$ The proper function of endothelial cells is very easily disturbed by nonphysiologic (either too high or too low) WSS. ${ }^{32-34}$

In our series, sIAs that lacked an intact endothelium had more inflammation, and lack of an endothelium was associated with flow conditions similar to those of inflammation. These similar associations among flow conditions, wall inflammation, and loss of endothelial cells suggest that endothelial damage caused by nonphysiologic flow conditions can be a mechanism by which flow conditions trigger or modulate inflammation in the sIA wall.

Although this is the first study to demonstrate associations with sIA wall degeneration and flow conditions in vivo in true sIAs in patients, aberrant flow conditions have been suspected as promoters of sIA wall degeneration, growth, and rupture before. ${ }^{10,16,31}$ Loss of an intact endothelium is characteristic of ruptured sIAs and of degenerated sIA walls. ${ }^{5,6}$ Loss of endothelium exposes thrombogenic matrix surfaces ${ }^{35}$ and thus predisposes to thrombus formation, which is also characteristic of ruptured sIAs and of degenerated sIA walls. ${ }^{5,6}$ Thrombus can trigger and promote wall degeneration via several mechanisms that cause cell death and proteolytic injury. ${ }^{10,16,38}$ In this series, high inflammation $(\mathrm{CD} 45+)$ was found in sIAs with organized thrombus (older thrombus) but not in sIAs with only fresh thrombus, suggesting that the prolonged presence of thrombus or degradation of the thrombus with time is what increases inflammation in the sIA wall.

\section{Clinical Implications}

Both high and low WSS have previously been implicated as promoters of sIA wall degeneration. ${ }^{12-14,39}$ Most interesting, in this series, high WSS and conditions of high flow in general (high shear rate, high vorticity, high viscous dissipation) were associated with inflammation, whereas low flow was associated with a degenerated wall that had lost mural cells. The observation that different types of flow were associated with distinct histologic changes suggests that flow may affect sIA wall remodeling via several mechanisms and that flow conditions can induce different changes in the sIA wall at different time points during the remodeling of the sIA wall. ${ }^{40}$ 


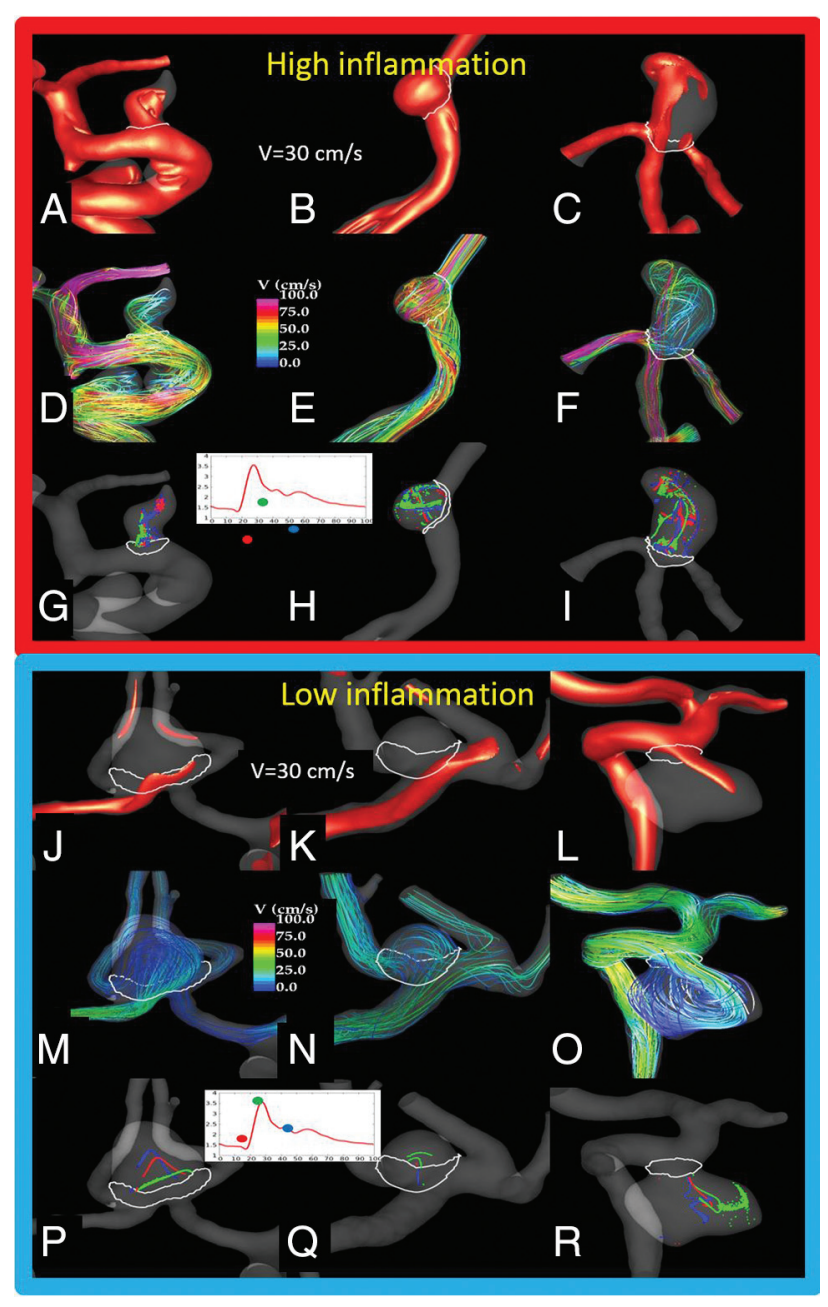

FIG 4. Flow conditions in the 6 sample aneurysms (Fig 3) with high or no inflammation in the wall. The visualizations show the following: the inflow jet at peak systole $(A-C, J-L)$, the flow pattern at peak systole $(D-F, M-O)$, and vortex core lines at 3 instances during the cardiac cycle $(G-I, P-R)$, to illustrate both the complexity of the flow pattern and its change during the cycle (instability). An aneurysm with inflammation (upper panel) had a strong inflow jet impacting the aneurysm dome and producing complex (but somewhat stable) flow structures and associated WSS distributions with regions of high WSS and regions of low WSS near the outflow. In contrast, aneurysms with no inflammation had more diffuse inflows entering the aneurysms at lower velocity and diffusing into a simpler but more variable (unstable) flow pattern, with associated WSS distribution characterized by a fairly uniformly low WSS.

Although it is still unclear whether inflammation is the cause of sIA rupture or a reaction to the cause of the degenerative remodeling that predisposes to rupture, inflammation is clearly an indicator of a degenerated, rupture-prone sIA wall, as demonstrated by histopathologic studies ${ }^{5,6,28}$ and by ferumoxytol-based MR imaging of inflammatory cells in the sIA wall in patients. ${ }^{41}$ We now demonstrate that flow conditions determined with patient-specific computational fluid dynamics simulations are associated with the presence of inflammation in the sIA wall. This finding supports the concept that patient-specific flow simulations could be used to distinguish those sIAs that will develop wall inflammation and thus would require either more frequent follow-up imaging or perhaps even more active treatment. Most important, our results also show that computerized geometric indexes are not similarly associated with wall inflammation and cannot replace flow simulations, even if sIA and parent artery geometry are some of the main determinants of flow conditions in the aneurysm.

In this series, flow dynamics was associated with wall inflammation and with other histologic changes in the sIA wall in small sIAs. Because it is well-established that risk of sIA rupture increases with sIA size $e^{42,43}$ but that many of the sIAs that do rupture are small, ${ }^{44}$ it is particularly useful to develop novel diagnostic tools that would predict rupture risk in small sIAs that otherwise would be mistaken for benign, low risk lesions.

\section{Limitations of the Study and Topics for Further Studies}

Our series is a highly selected one, with bias in sIA size and clinical risk factors. This kind of selection bias is unavoidable in any kind of study that uses patient-derived tissue samples collected intraoperatively. Moreover, because not all sIAs undergo an operation and not all of those that do are suitable for the acquisition of tissue samples, our series is somewhat limited in number of samples and statistical power. Computational fluid dynamics models make several assumptions and are constructed from CTA images with limited resolution, which could affect the representation of the intrasaccular flows, especially for aneurysms with narrow necks. Our findings need to be replicated in other, similar or larger patient series and in experimental models. Experimental models and computer simulations with cell-cell interaction are needed to establish causality between the different associations and to provide mechanistic insight.

Most interesting, in this series that focused on the time- and space-averaged flow conditions in the whole sIA, heterogeneity in the distribution of WSS across the aneurysm sac was associated with heterogeneity in the number of CD $45+$ cells in the wall. This finding suggests that the flow-induced cellular interactions that lead to increased inflammation (eg, loss of endothelial cells) might happen focally at the wall. Further studies should include histologic data from different regions of the sIA wall and spatial registration of these histologic changes with focal flow conditions. These studies are also needed to confirm our findings because sIA walls are highly heterogeneous in structure (Fig 1) and this local variation in the aneurysm wall may have biased our results because we were not able to analyze the histology of the whole sIA wall.

In addition, flow simulations need to be correlated with the biology of the sIA wall and the structural strength of the aneurysm wall, to develop a diagnostic tool from computational fluid dynamics models. ${ }^{8,35}$ This requirement is paramount to understand how different kinds of flow patterns affect the sIA wall, which is critical to properly use this technology in diagnostics.

\section{CONCLUSIONS}

We show that flow conditions in saccular intracranial aneurysms are associated with inflammation and other histologic changes in the aneurysm wall. In particular, high- and low-flow conditions are associated with different wall changes. This study suggests that computational simulations that determine patient-specific flow conditions could be used to identify aneurysms at risk for devel- 
oping inflammation and other rupture-associated changes in their walls.

Degenerative wall remodeling, inflammation, and nonphysiologic flow conditions have been previously shown to be associated with sIA rupture. ${ }^{6,13,14,28}$ Although we and others have suggested possible mechanisms by which pathologic flow conditions may lead to degenerative wall remodeling, ${ }^{10,12,16,29}$ this is the first study that compares in vivo flow conditions with the histology of human intracranial aneurysms.

Disclosures: Juan Cebral-RELATED: Grant: National Institutes of Health*; UNRELATED: Grants/Grants Pending: National Institutes of Health, Philips Healthcare. Eliisa Ollikainen-RELATED: Grant: Helsinki University Central Hospital Engineering Virtual Organization grants, Helsinki Biomedical Graduate Program Funding, Orion Research Foundation, and the Finnish Medical Foundation; UNRELATED: Employment: Helsinki City Hospital and Helsinki University Central Hospital. Fernando Mut-RELATED: Grant: National Institutes of Health.* Visa Sippola-UNRELATED: Employment: Helsinki and Uusimaa Hospital District, Aalto University, Comments: employment as a researcher, no conflicts of interest concerning the study topic. Mika Niemelä—RELATED: TYH2014236, ${ }^{*}$ Comments: research grant by the state. Anne Robertson-RELATED: Grant: National Institutes of Health National Institute of Neurological Disorders and Stroke (R21NS080031).* Juhana Frösen—RELATED:

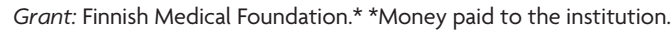

\section{REFERENCES}

1. Vlak MH, Algra A, Brandenburg R, et al. Prevalence of unruptured intracranial aneurysms, with emphasis on sex, age, comorbidity, country, and time period: a systematic review and meta-analysis. Lancet Neurol 2011;10:626-36 CrossRef Medline

2. Karamanakos PN, von Und Zu Fraunberg M, Bendel S, et al. Risk factors for three phases of 12-month mortality in 1657 patients from a defined population after acute aneurysmal subarachnoid hemorrhage. World Neurosurg 2012;78:631-39 CrossRef Medline

3. Korja M, Lehto H, Juvela S. Lifelong rupture risk of intracranial aneurysms depends on risk factors: a prospective Finnish cohort study. Stroke 2014;45:1958-63 CrossRef Medline

4. Kotowski M, Naggara O, Darsaut TE, et al. Safety and occlusion rates of surgical treatment of unruptured intracranial aneurysms: a systematic review and meta-analysis of the literature from 1990 to 2011. J Neurol Neurosurg Psychiatry 2013;84:42-48 CrossRef Medline

5. Kataoka K, Taneda M, Asai T, et al. Structural fragility and inflammatory response of ruptured cerebral aneurysms: a comparative study between ruptured and unruptured cerebral aneurysms. Stroke 1999;30:1396-401 CrossRef Medline

6. Frösen J, Piippo A, Paetau A, et al. Remodeling of saccular cerebral artery aneurysm wall is associated with rupture: histological analysis of 24 unruptured and 42 ruptured cases. Stroke 2004;35:2287-93 CrossRef Medline

7. Tulamo R, Frösen J, Hernesniemi J, et al. Inflammatory changes in the aneurysm wall: a review. J Neurointerv Surg 2010;2:120-30 CrossRef Medline

8. Robertson AM, Duan X, Aziz KM, et al. Diversity in the strength and structure of unruptured cerebral aneurysms. Ann Biomed Eng 2015; 43:1502-15 CrossRef Medline

9. Marbacher S, Marjamaa J, Bradacova K, et al. Loss of mural cells leads to wall degeneration, aneurysm growth, and eventual rupture in a rat aneurysm model. Stroke 2014;45:248-54 CrossRef Medline

10. Frösen J, Tulamo R, Paetau A, et al. Saccular intracranial aneurysm: pathology and mechanisms. Acta Neuropathol 2012;123:773-86 CrossRef Medline

11. Sforza D, Putman CM, Cebral JR. Hemodynamics of cerebral aneurysms. Annu Rev Fluid Mech 2009;41:91-107 CrossRef Medline

12. Shojima M, Oshima M, Takagi $K$, et al. Magnitude and role of wall shear stress on cerebral aneurysm: computational fluid dynamic study of $\mathbf{2 0}$ middle cerebral artery aneurysms. Stroke 2004;35: 2500-05 CrossRef Medline

13. Cebral JR, Mut F, Weir J, et al. Quantitative characterization of the hemodynamic environment in ruptured and unruptured brain aneurysms. AJNR Am J Neuroradiol 2011;32:145-51 CrossRef Medline

14. Xiang J, Natarajan SK, Tremmel M, et al. Hemodynamic-morphologic discriminants for intracranial aneurysm rupture. Stroke 2011; 42:144-52 CrossRef Medline

15. Castro MA, Putman CM, Radaelli A, et al. Hemodynamics and rupture of terminal cerebral aneurysms. Acad Radiol 2009;16:1201-07 CrossRef Medline

16. Ujiie $\mathrm{H}$, Tachibana $\mathrm{H}$, Hiramatsu $\mathrm{O}$, et al. Effects of size and shape (aspect ratio) on the hemodynamics of saccular aneurysms: a possible index for surgical treatment of intracranial aneurysms. Neurosurgery 1999;45:119-29; discussion 129-30 CrossRef Medline

17. Ollikainen E, Tulamo R, Frösen J, et al. Mast cells, neovascularization, and microhemorrhages are associated with saccular intracranial artery aneurysm wall remodeling. J Neuropathol Exp Neurol 2014;73:855-64 CrossRef Medline

18. Cebral JR, Castro MA, Appanaboyina S, et al. Efficient pipeline for image-based patient-specific analysis of cerebral aneurysm hemodynamics: technique and sensitivity. IEEE Trans Med Imaging 2005;24:457-67 CrossRef Medline

19. Mut F, Aubry R, Löhner R, et al. Fast numerical solutions of patientspecific blood flows in 3D arterial systems. Int J Num Meth Biomed Eng 2010;26:73-85 CrossRef Medline

20. Taylor CA, Hughes TJ, Zarins CK. Finite element modeling of blood flow in arteries. Comp Meth App Mech Eng 1998;158:155-96 CrossRef

21. Ford MD, Alperin N, Lee SH, et al. Characterization of volumetric flow rate waveforms in the normal internal carotid and vertebral arteries. Physiol Meas 2005;26:477-88 CrossRef Medline

22. Cebral JR, Castro MA, Putman CM, et al. Flow-area relationship in internal carotid and vertebral arteries. Physiol Meas 2008;29:585-94 CrossRef Medline

23. Murray CD. The physiological principle of minimum work, II: oxygen exchange in capillaries. Proc Natl Acad Sci U S A 1926:12: 207-14 Medline

24. Mut F, Löhner R, Chien A, et al. Computational hemodynamics framework for the analysis of cerebral aneurysms. Int J Numer Method Biomed Eng 2011;27:822-39 CrossRef Medline

25. Cebral JR, Duan X, Gade PS, et al. Regional mapping of flow and wall characteristics of intracranial aneurysms. Ann Biomed Eng 2016 Jun 27. [Epub ahead of print] Medline

26. Ma B, Harbaugh RE, Raghavan ML. Three-dimensional geometrical characterization of cerebral aneurysms. Ann Biomed Eng 2004;32: 264-73 CrossRef Medline

27. Raghavan ML, Ma B, Harbaugh RE. Quantified aneurysm shape and rupture risk. J Neurosurg 2005;102:355-62 CrossRef Medline

28. Tulamo R, Frösen J, Junnikkala S, et al. Complement activation associates with saccular cerebral artery aneurysm wall degeneration and rupture. Neurosurgery 2006;59:1069-76; discussion 1076-77 Medline

29. Cebral JR, Castro MA, Burgess JE, et al. Characterization of cerebral aneurysms for assessing risk of rupture by using patient-specific computational hemodynamics models. AJNR Am J Neuroradiol 2005;26:2550-59 Medline

30. Frösen J. Smooth muscle cells and the formation, degeneration, and rupture of saccular intracranial aneurysm wall: a review of current pathophysiological knowledge. Transl Stroke Res 2014;5:347-56 CrossRef Medline

31. Chalouhi N, Hoh BL, Hasan D. Review of cerebral aneurysm formation, growth, and rupture. Stroke 2013;44:3613-22 CrossRef Medline

32. Tarbell JM, Simon SI, Curry FR. Mechanosensing at the vascular interface. Annu Rev Biomed Eng 2014;16:505-32 CrossRef Medline

33. Tarbell JM. Shear stress and the endothelial transport barrier. Cardiovasc Res 2010;87:320-30 CrossRef Medline

34. Pan S. Molecular mechanisms responsible for the atheroprotective effects of laminar shear stress. Antioxid Redox Signal 2009;11: 1669-82 CrossRef Medline 
35. Aksu K, Donmez A, Keser G. Inflammation-induced thrombosis: mechanisms, disease associations and management. Curr Pharm Des 2012;18:1478-93 CrossRef Medline

36. Cebral JR, Duan X, Chung BJ, et al. Wall mechanical properties and hemodynamics of unruptured intracranial aneurysms. AJNR Am J Neuroradiol 2015;36:1695-703 CrossRef Medline

37. Kallmes DF. Point: CFD—computational fluid dynamics or confounding factor dissemination. AJNR Am J Neuroradiol 2012;33: 396-98 CrossRef Medline

38. Cebral JR, Meng H. Counterpoint: realizing the clinical utility of computational fluid dynamics-closing the gap. AJNR Am J Neuroradiol 2012;33:396-98 CrossRef Medline

39. Meng H, Tutino VM, Xiang J, et al. High WSS or low WSS? Complex interactions of hemodynamics with intracranial aneurysm initiation, growth, and rupture-toward a unifying hypothesis. AJNR Am J Neuroradiol 2014;35:1254-62 CrossRef Medline

40. Robertson AM, Watton PN, Computational fluid dynamics in an- eurysm research: critical reflections, future directions. AJNR Am J Neuroradiol 2012;33:992-95 CrossRef Medline

41. Hasan D, Chalouhi N, Jabbour P, et al. Early change in ferumoxytol-enhanced magnetic resonance imaging signal suggests unstable human cerebral aneurysm: a pilot study. Stroke 2012;43:3258-65 CrossRef Medline

42. Wiebers DO, Whisnant JP, Huston J 3rd, et al; International Study of Unruptured Intracranial Aneurysms Investigators. Unruptured intracranial aneurysms: natural history, clinical outcome, and risks of surgical and endovascular treatment. Lancet 2003;362:103-10 CrossRef Medline

43. Morita A, Kirino T, Hashi K, et al; UCAS Japan Investigators. The natural course of unruptured cerebral aneurysms in a Japanese cohort. N Engl J Med 2012;366:2474-82 CrossRef Medline

44. Huttunen T, von und zu Fraunberg M, Frösen J, et al. Saccular intracranial aneurysm disease: distribution of site, size, and age suggests different etiologies for aneurysm formation and rupture in 316 familial and 1454 sporadic eastern Finnish patients. Neurosurgery 2010;66:631-38; discussion 638 CrossRef Medline 